\title{
Analysis of PCM Material in Thermal Energy Storage System
}

\author{
Amrit Om Nayak, M.Gowtham, R.Vinod, and G.Ramkumar
}

\begin{abstract}
A phase-change material (PCM) is a substance with a high latent heat storage capacity which on melting and solidifying at a certain temperature, is capable of storing and releasing large amounts of energy. Various PCM like Paraffin wax, sodium acetate tri-hydrate and phenolphthalein are considered which are used to absorb heat from the coolant water from the engine. The conduction and convection criterion of heat transfer enable the PCM to store this heat as latent heat. The amount of convection and temperature change brought about due to the heat flux has been simulated and studied in detail using GAMBIT and FLUENT .
\end{abstract}

Index Terms - Latent Heat storage capacity, phase change materials, paraffin wax, sodium acetate tri-hydrate, phenolphthalein

\section{INTRODUCTION}

Increasing environmental pollution is an important problem observed during cold start of internal combustion engines. Cold start of internal combustion engines is characterized by various problems such as increase of fuel consumption resulting from heterogonous combustion, increasing concentration of toxic emissions, increase of lubricant viscosity and resistance to motion and thereby increase of load on the accumulator and starter resulting in increased vibration and noise. Developments of new devices that solve this problem are a necessity. Thermal energy storage system (TESS) is one such device. The TESS uses Phase Change Material's (PCM) latent heat storage capacity for pre-heating the internal combustion engine. The thermal energy storage device (TESD) works on the effect of absorption and rejection of heat during the solid-liquid phase change of heat storage material. The overall function of the TESS is dominated by the PCM. The PCM material should be selected considering the application and the working conditions. Depending on the applications, the PCMs should first be selected based on their melting temperature. Materials that melt below $288 \mathrm{~K}$ are used for storing coolness in air conditioning applications, while materials that melt above $363 \mathrm{~K}$ are used for absorption refrigeration. All other materials that melt between these two temperatures can be applied in solar heating and for heat load leveling applications. Gu et al [1] developed a heat recovery system using PCM to recover the rejected heat of air conditioning systems and produce low temperature hot water for washing and bathing. They concluded that the heat recovery system

Manuscript received November 4, 2011; revised December 9, 2011.

Authors are with Department of Mechanical Engineering, Thiagarajar College of Engineering, Madurai, India (email: amritomnayak@gmail.com; gowthamtce@gmail.com; ramtcemech09@gmail.com). decreases not only the consumption of primary energy for heating domestic hot water but also the calefaction to the surroundings due to the rejection of heat from air conditioning systems. Hong et al [2] tried to improve the accuracy of the T-history method for measuring heat of fusion of various materials and many of the PCMs were taken under study. Duffy [3] proposed a numerical simulation of porous latent heat thermal energy storage device for thermoelectric cooling under different porosities of the aluminum matrix. They used a porous aluminum matrix as a way of improving the performance of the system, enhancing heat conduction without reducing significantly the stored energy. Ravikumar et al [4] has proposed Cool storage system using phase change materials can be used for peak load shifting. Regin et al [5] proposed a paper in analyzing the behavior of a packed bed latent heat thermal energy storage system. The packed bed is composed of spherical capsules filled with paraffin wax as PCM usable with a solar water heating system and the phase change phenomena of PCM inside the capsules are analyzed by using enthalpy method. The equations are numerically solved, and the results obtained are used for the thermal performance analysis of both charging and discharging processes. Mahmud et al [6] studied a theoretical investigation of thermal and physical properties of a phase change material which consists of paraffin wax with 5\% aluminum powder, this composite used as a thermal storage system for solar applications Demirbas et al [7] studied about thermal energy storage materials and phase change materials and concluded that paraffin waxes are cheap and have moderate TES density but low thermal conductivity and, hence, require a large surface area. Hydrated salts have a larger energy storage density and a higher thermal conductivity. Amin et al [8] proposed and optimized usage of PCMs encapsulated in slabs. Using a validated numerical model of the system, a parametric study was undertaken to investigate the impact of the slab thickness, gap between slabs and the mass flow rate. Sari et al [9] studied determination of proper amount of paraffin absorbed into expanded graphite to obtain form-stable composite as phase change material. Zhang et al [10] found that expanded graphite composite phase change material had a large thermal storage capacity and improved thermal conductivity and did not experience liquid leakage during its solid-liquid phase change. He obtained a thermal conductivity which was higher than that of the paraffin, due to the combination with the expanded graphite that had a high thermal conductivity.

Phase change material storages are used to balance temporary temperature alternations and to store energy in several practical application areas. PCM storage is preferable 
to sensible heat storage in applications with a small temperature swing because of its nearly isothermal storing mechanism and high storage density. Phase change materials are employed in different fields of thermal engineering like energy storage, thermal conditioning of buildings, waste heat recovery, off peak power utilization, heat pump systems, space applications, laptop computer cooling, and telecom shelters. When a temperature peak occurs, PCM absorbs the excessive energy by going through a phase transition and releasing the absorbed energy later when the peak has passed off.

The possibility of varying conditions during the design process urges us to design a reliable PCM model which would enable parametric studies to be conducted at speed and would also enable the comparison of several alternatives. This would also preclude the need to build experimental platforms for measurements.

\section{THEORY}

Heat transfer in PCM storage is characterized by a moving solid-liquid interface, generally referred to as the "moving boundary" problem. It is a transient, non-linear phenomenon. Analytical solutions for phase change problems are only known for a couple of physical situations which possess a simple geometry and simple boundary conditions, as nonlinearity poses major difficulties in moving boundary problems. Neumann originated the most well-known precise analytical solution for a one-dimensional moving boundary problem, called the Stefan problem. Some analytical approximations for one-dimensional moving boundary problems with different boundary conditions are the quasi-stationary approximation, perturbation methods, the Megerlin method and the Heat-balance-integral method. It has been assumed here that the melting or solidification temperature is constant. However, for example technical grade paraffin has a wide temperature range at the points where melting and solidification occur.

Phase change problems are usually solved with finite difference or finite element methods in accordance with the numerical approach. The phase change phenomenon has to be modeled separately due the non-linear nature of the problem. A wide range of different kinds of numerical methods for solving PCM problems exist. The most common methods used are the enthalpy method and the effective heat capacity method.

\section{Thermal EnERgy Storage Systems (TeSs)}

The demand and supply gap for energy sources is widening day by day. Moreover, the fact that the energy can neither be created and nor destroyed has resulted in focusing of scientific research in the direction of storing the different forms of energy using diverse devices. Thermal energy is one such energy which is of interest to researchers worldwide. Thermal energy could have several geneses but storage of solar thermal energy is one of the principal areas of investigation. In recent years, various conventional and unconventional materials are investigated for their capability to store thermal energy. These thermal energy storage devices (TESD) are selected on the basis of some crucial physical, chemical and economic properties. Melting point, heat of fusion, density, heat capacity, thermal conductivity, compatibility with container and cost of production are the chief parameters for selection of TESD. It is a genuine challenge to find out an ultimate TESD as the overall suitability of materials to be used as TESD is governed by a multifaceted interplay between several properties of those materials.

\section{Properties AfFeCting Tess}

\section{A. Melting Point}

Melting point is the temperature at which the first crystal of the material collapses. It is imperative to have the melting point of TESD within the temperature range of application. The melting point as such does not affect the energy storage capacity of a material. However, as a phase change is involved in melting, the inclusion of melting point in temperature range of application can permit the use of phase change as an on-off switch. Melting point lower as well as higher than the temperature range of application prohibits the use of the material in TESD.

\section{B. Heat of Fusion $(\Delta H)$}

Heat of fusion $(\Delta \mathrm{H})$ also known as enthalpy of fusion or latent heat of fusion is a very important property useful in selecting a TESD. It refers to the amount of thermal energy that a material must absorb or evolve in order to change its phase from solid to liquid or vice versa. Large values of heat of fusion aid in increasing the efficiency of TESDs.

\section{Heat Capacity}

Heat capacity refers to the amount of energy per molecule that a compound can store before the increase in its temperature. This energy is generally stored in translational, vibration and rotational modes. Thus materials with greater number of atoms in its composition are expected to have higher heat capacity.

\section{Thermal Conductivity $(K)$}

Thermal Conductivity (k) measures the ability of a material to conduct heat. Greater values of $\mathrm{k}$ imply an efficient heat transfer. Thermal conductivity is a property which needs to be optimized. Since thermal conductivity is phase dependant property, it is important to know values of $\mathrm{k}$ in both the solid as well as molten phases. It has been observed that most of molten materials exhibit much higher values of thermal conductivity as compared to that in their solid state. Higher values of thermal conductivity in molten state can facilitate an efficient heat transfer and smooth operation of the thermal circuits in TESD.

\section{E. Density $(\rho)$}

Density $(\rho)$ of a material refers to its mass per unit volume. Density values can readily be measured using densitometers. Materials with higher density thus occupy less space which in turn increases the energy storage capacity. Materials with high density obviously possess higher energy storage capacity but many of them show a significant decrease in density in their molten state. This is due to the expansion of 
their volume. Thus, if the TESD is placed in a sealed container in its solid phase, empty space equivalent to its volume expansion must be kept in the container. Considering the above, it can be generalized that materials with high density but very small change in density at the phase change temperature are attractive TESDs.

\section{F. Required Properties of a PCM}

- High latent heat of fusion per unit mass, so that a lesser amount of material stores a given amount of energy.

- High specific heat that provides additional sensible heat storage effect and also avoid sub cooling

- High density, so that a smaller container can hold the material.

- A melting point in the desired operating temperature range.

- The phase change material should be non-poisonous, non-flammable and non-explosive.

- No chemical decomposition, so that the (TESS) system life is assured.

- PCM should exhibit little or no super cooling during freezing.

- No corrosiveness to construction material.

To solve some of the problems inherent in inorganic PCMs, interest has to be tuned towards a new class of material substances such as paraffins, fatty acids and polyethylene glycol. Those materials were more costly than common salt hydrates and they have somewhat lower heat storage capacity per unit volume.

\section{Proposed Model}

A mechanical set up of a coolant box with an inlet and outlet pipe is taken. The PCM material is filled inside the PCM container which is placed in the middle of the coolant heat exchanger as in Fig.1. The coolant water that flows round the engine enters the coolant box with a velocity (V1) temperature (T1) and at a pressure (P1). The coolant water transfers heat within itself to attain an equilibrium temperature as a result of the convection. Conduction of heat takes place at the interface of coolant water and the PCM container which in turn conducts the heat to the PCM material. The PCM material now absorbs heat till its latent heat of fusion is reached. Phase change occurs now. After the phase change occurs, convection of heat takes place within the melted PCM till equilibrium is reached. The heat stored within the PCM can be later on used in purpose of heating up of the engine again while starting up initially.

\section{A. Location of TESD}

The basic scheme of the TESD connection to the cooling system of the engine is in Fig.2. The TESD can be connected to the engine water jacket. During charge period, liquid moves through the TESD, the water jacket of the engine and radiator with the help of the water pump of engine. During discharging, because the thermostat is closed cooling liquid moves through only the TESD and the water jacket of the engine with the help of the electrical pump. The TESS for preheating of the engine consists of the TESD.

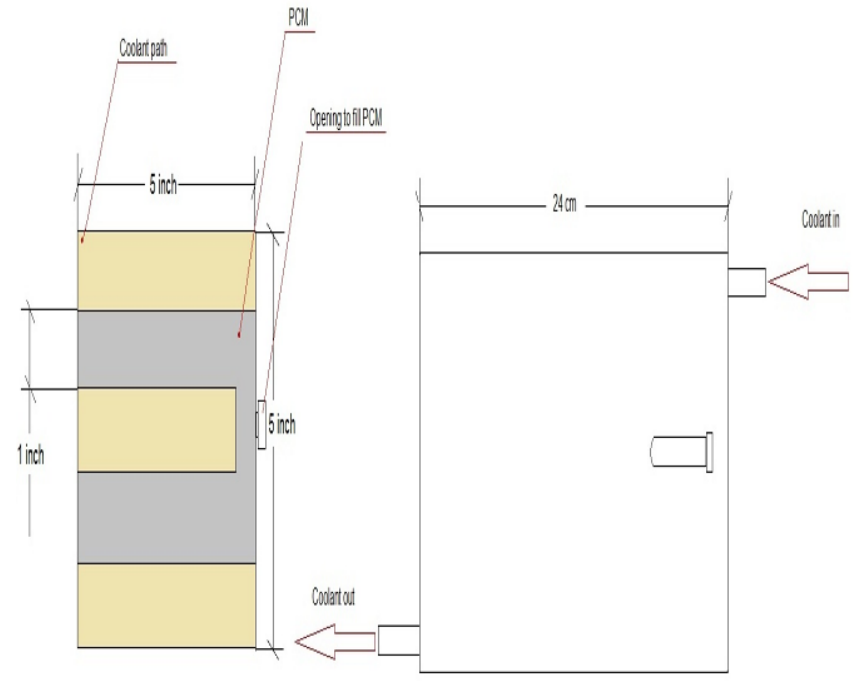

Fig. 1. Schematic view of the TESD

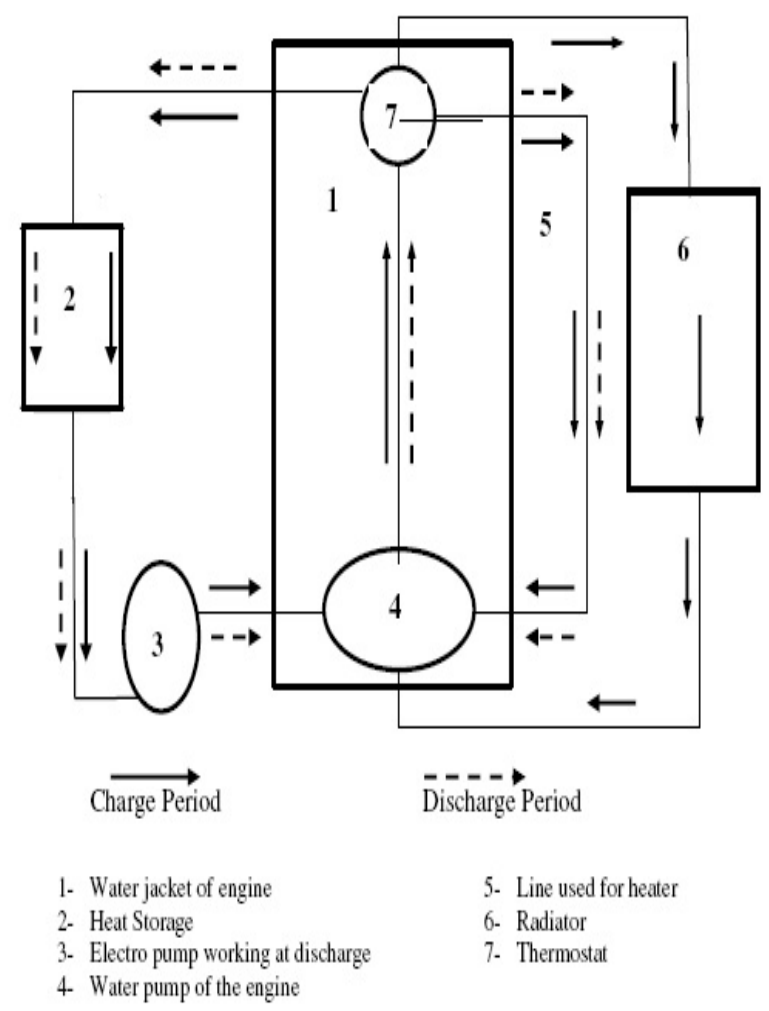

Fig 2. Position of TESD in Engine System

\section{B. Grid Generation and Numerical Solutions by Fluent 6.2}

CFD techniques consist of numerical solutions of mass, Momentum and energy conservation with other equations like species transport. Solution to CFD problems is obtained in two main stages. The PCM volume is divided into small control volumes called mesh in the first stage. The structure of the TESD is created with the PCM storage tank inside it. Using Boolean operations of subtraction in Gambit, we subtracted the PCM volume from the TESD volume. This volume was meshed as shown in Fig.3. 


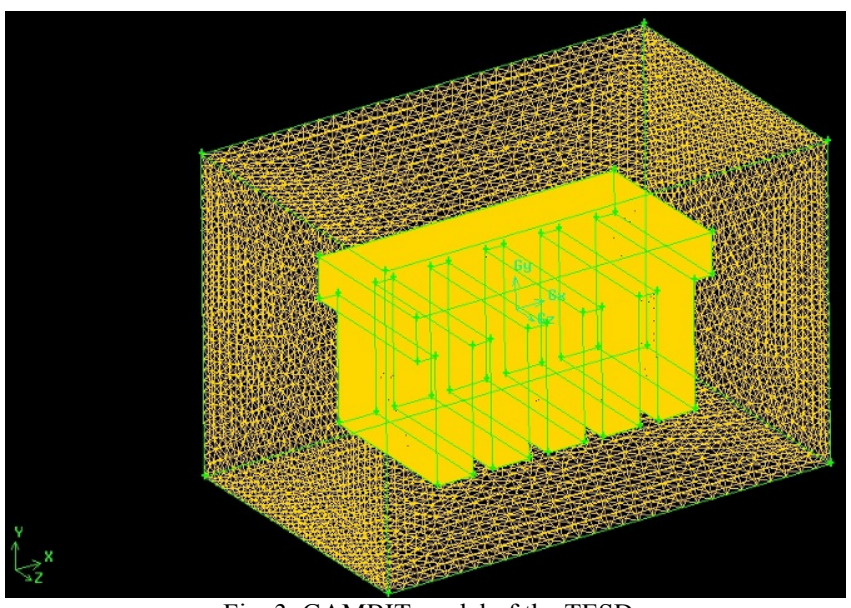

Fig. 3. GAMBIT model of the TESD

The PCM volume was recreated and meshed separately. This allowed us to obtain the exact volumes of the TESD and PCM container for meshing purposes. Next part is the numerical solutions of mass, Momentum and energy conservation equations. In this case, unsteady energy balance equation has been solved in solid phase and unsteady momentum and energy balance equation have been solved simultaneously in liquid phase for each control volume. After making intricate meshing in gambit, it is exported to Fluent and in $3 \mathrm{D}$, simulations were made using the material properties in Table I.

TABLE I: PROPERTIES OF PCM MATERIALS

\begin{tabular}{|c|c|c|c|}
\hline \multirow{2}{*}{ Physical Parameters } & \multicolumn{3}{|c|}{ Phase Change Material } \\
\cline { 2 - 4 } & $\begin{array}{c}\text { Paraffin } \\
\text { Wax }\end{array}$ & $\begin{array}{c}\text { Sodium Acetate } \\
\text { Tri-hydrate }\end{array}$ & Naphthalene \\
\hline $\begin{array}{c}\text { Wall Temperature in } \\
\text { Kelvin (K) }\end{array}$ & 343 & 343 & 343 \\
\hline Viscosity (kg/m.s) & 1.90 & $-^{*}$ & $-^{*}$ \\
\hline Density (kg/m $\mathrm{m}^{3}$ & 900 & 1450 & 1140 \\
\hline $\begin{array}{c}\text { Heat conduction } \\
(\mathrm{W} / \mathrm{m} . \mathrm{K})\end{array}$ & 0.24 & 0.5 & 0.1221 \\
\hline $\begin{array}{c}\text { Specific heat capacity } \\
(\mathrm{C})\end{array}$ & 2900 & 1088 & 1720 \\
$(\mathrm{~J} / \mathrm{kg} . \mathrm{K})$ & & & \\
\hline
\end{tabular}

NOTE*: These values of viscosity are not required as sodium acetate trihydrate doesn't melt and naphthalene sublimes.

\section{RESUlts AND DisCUSSION}

The transient temperature profiles have been obtained by solving unsteady energy balance equation using Fluent 6.2 version. The temperatures profiles have been estimated. As the natural convection was considered in liquid phase therefore, unsteady momentum and energy balance equation have been solved simultaneously in liquid phase.

\section{A. Results Obtained for Paraffin Wax}

The temperature contours in Fig. 4 and Fig. 5 clearly show the decrease in temperature of the coolant water from $343 \mathrm{~K}$ to $332 \mathrm{~K}$. The PCM exhibits a temperature change of almost $16 \mathrm{~K}$ as observed from the above graphical images obtained through fluent analysis.

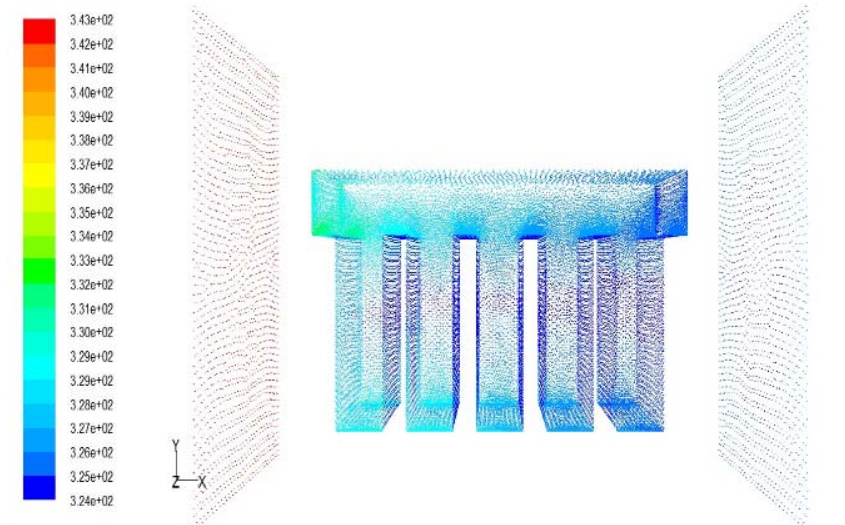

Velocity Vectors Colored By Static Temperature (k) (Time $=0.0000 \mathrm{e}+00)$ FLUENT 6.2 (3d, segregated, am 25, 201

Fig. 4. Temperature Contours of Paraffin wax

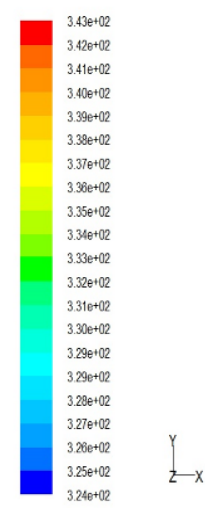

Velocity Vectors Colored By Static Temperature $(\mathrm{k})($ Time $=0.0000 \mathrm{e}+00)$ FLUENT 6.2 (3d, segregated, lam, unsteady)

Fig. 5. Temperature Contours of TESD containing Paraffin wax

\section{B. Results Obtained for Sodium Acetate Tri-Hydrate}

We observe that the temperature contours in Fig. 6 and Fig. 7 clearly show the decrease in temperature of the coolant water from $343 \mathrm{~K}$ to $324 \mathrm{~K}$. The PCM exhibits a temperature change of almost $25 \mathrm{~K}$. as observed from the above graphical images obtained through fluent analysis.

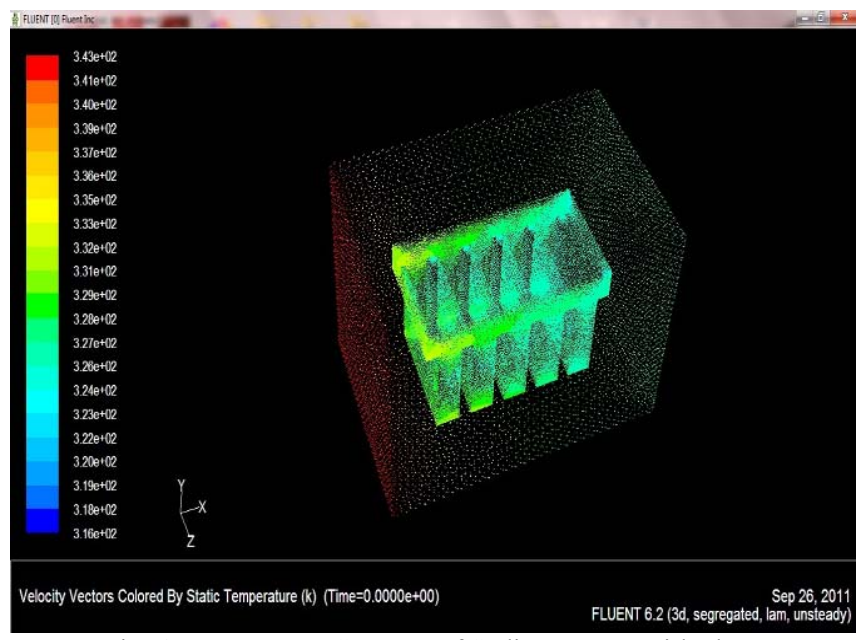

Fig. 6. Temperature Contours of Sodium acetate tri-hydrate 


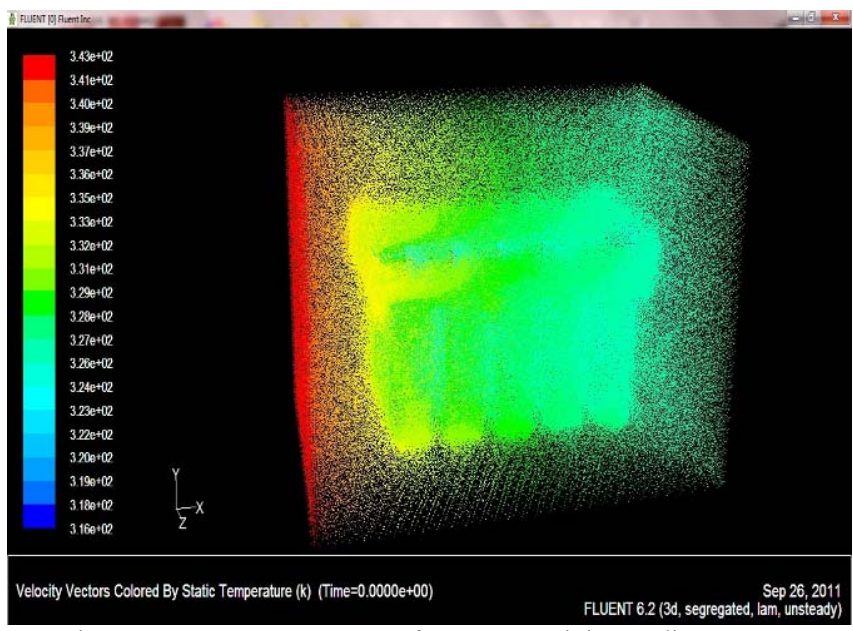

Fig. 7. Temperature Contours of TESD containing Sodium acetate tri-hydrate

\section{Results obtained for Naphthalene}

The temperature contours in Fig. 8 and Fig. 9 clearly show the decrease in temperature of the coolant water from $343 \mathrm{~K}$ to $327 \mathrm{~K}$. The PCM exhibits a temperature change of almost $20 \mathrm{~K}$. as observed from the above graphical images obtained through fluent analysis.

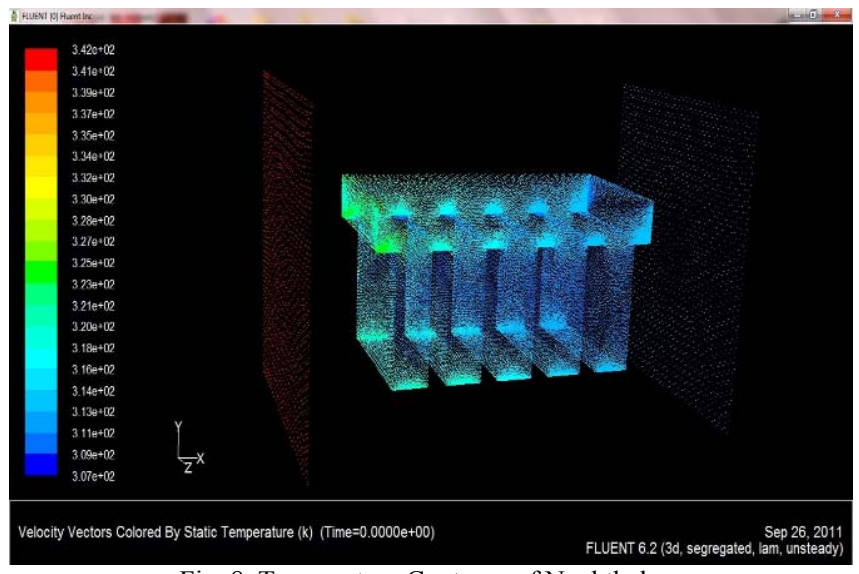

Fig. 8. Temperature Contours of Naphthalene

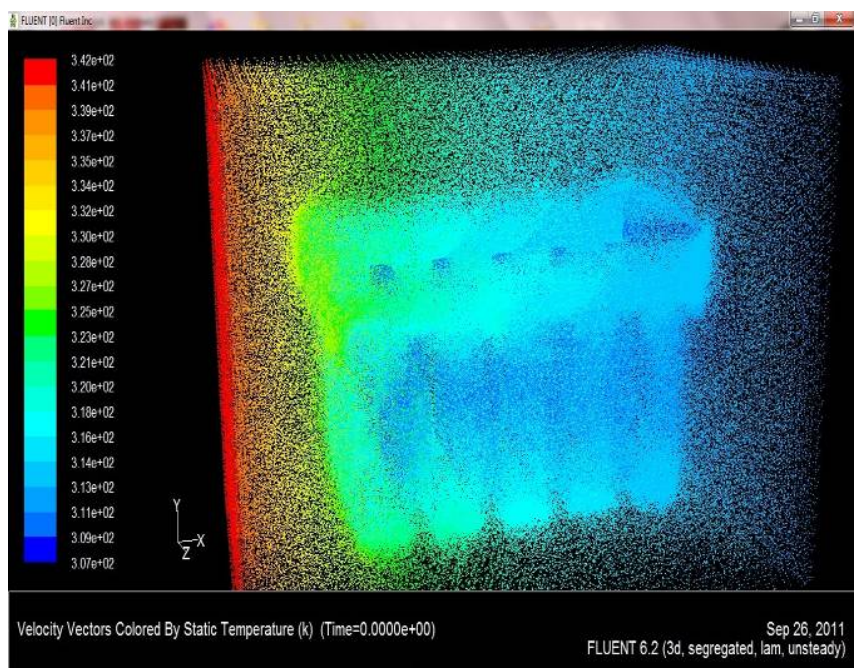

Fig. 9. Temperature Contours of TESD containing Naphthalene

\section{CONCLUSION}

Successful analysis of the transient heat transfer characteristics of phase change material has been studied. From the temperature profiles we observe that sodium acetate tri-hydrate gives us the most promising results when compared to paraffin wax and naphthalene. Coolant water loses maximum heat to sodium acetate tri-hydrate which is obtained as drop in temperature from $343 \mathrm{~K}$ to $324 \mathrm{~K}$ (in the coolant water) from the graphs obtained. It is observed that the heat absorption in the PCM material decreases gradually as we travel from the inlet of coolant water towards the outlet of coolant water. The phase change near wall is noted by the formation of a flat plateau regime on temperature vs. time plot. Different contours were generated by using Fluent. The temperatures profiles have been estimated.

\section{REFERENCES}

[1] Gu, Z., Liu, H., Li, Y. Thermal energy recovery heat recovery system calculation and phase change material development. Applied Thermal Engineering 24: (2004) 2511-2426

[2] Hong, H., Kim, S.K., Kim, Y.S. Accuracy improvement of t-history method for measuring heat of fusion of various materials. International Journal of Refrigeration 27: (2004) 360-366

[3] Trelles, J.P., Duffy, J.P , Numerical simulation of porous latent heat thermal energy storage for thermoelectric cooling. Applied Thermal Engineering 23: (2003) 1647-1664.

[4] M.Ravikumar, PSS. Srinivasan, Phase change material as a thermal energy storage material for cooling of building, Journal of Theoretical and Applied Information Technology (2005) 503-512

[5] A. Felix Regin, S.C. Solanki, J.S. Saini, An analysis of a packed bed latent heat thermal energy storage system using PCM capsules: Numerical investigation, Renewable Energy 34 (2009) 1765-1773

[6] Alkilani Mahmud, Sopian K., Alghoul M. A.,Mat Sohif, Using a Paraffin wax-Aluminum compound as a thermal storage material in a solar air heater, ARPN Journal of Engineering and Applied Sciences, vol. 4, no. 10, December 2009

[7] Fatih demirbas, Thermal Energy Storage and Phase Change Materials: An Overview, Energy Sources, Part B, 1:85-95, 2006

[8] Nasrul Amri Mohd Amin, Martin Belusko, Frank Bruno, Optimisation of A Phase Change Thermal Storage System, World Academy of Science, Engineering and Technology 56 (2009)

[9] Sar1, Ali Karaipekli, Thermal conductivity and latent heat thermal energy storage characteristics of paraffin/expanded graphite composite as phase change material, Applied Thermal Engineering, Volume 27, Issues 8-9, June 2007, Pages 1271-1277

[10] Zhengguo Zhang, Xiaoming Fang , Study on paraffin/expanded graphite composite phase change thermal energy storage material, Energy Conversion and Management, Volume 47, Issue 3, February 2006, Pages 303-310 